\title{
Development of a new haptic perception instrument: a pilot study
}

\author{
Desenvolvimento de um novo instrumento de avaliação da percepção háptica: um \\ estudo piloto \\ Leonardo Penteado Nascimento', Joyce Martini', Mariana Callil Voos', Hsin Fen Chien², Fátima \\ Aparecida Caromano'
}

\begin{abstract}
Objective: Hand sensory tests do not consider distinct physiological receptors, nor detect normal range variations concerning developmental or pathological changes. We developed an instrument with a set of tests with timing and scoring for assessing haptic perception, which is the interaction between sensory and motor systems, in surfaces exploration, by moving hands. Method: Firstly, group meetings were set for test/manual conception and materials testing. The test/manual were submitted to 30 reviewers in 3 stages (10 reviewers on each stage). Results: The Hand Haptic Perception Instrument (HHPI) evaluates hand sensorimotor performance on six domains: depression, elevation, texture, compressibility, weight (barognosis) and form perception. Each domain requires specific materials. Score ranges from 0 to 57 , being 0 the worst rating. Conclusion: This methodological process allowed the development of six domains and instructions to assess haptic perception. This version of HHPI is a pilot model. Further studies will determine reliability and normality ranges.
\end{abstract}

Keywords: touch perception, form perception, weight perception, evaluation, hands.

\section{RESUMO}

Objetivo: Testes de sensibilidade manual não consideram receptores fisiológicos distintos, tampouco variações do desenvolvimento normal ou patológico. Desenvolvemos um instrumento, com pontuação e tempo de desempenho, para avaliar percepção háptica, que é a interação sensório-motora na exploração de superfícies, pelo movimento das mãos. Método: Reuniões de grupo foram estabelecidas para desenvolver os testes/manual e testar materiais. O instrumento e seu manual foram submetidos a 30 revisores, em 3 estágios (com 10 revisores em cada estágio). Resultados: 0 instrumento de avaliação da percepção háptica manual (IAPHM) avalia o desempenho sensório-motor da mão em seis domínios: depressão, elevação, textura, compressibilidade, peso (barognosia) e percepção de forma. Cada domínio requer materiais específicos. A pontuação vai de 0 a 57, sendo 0 a pior pontuação. Conclusão: Esse método permitiu o desenvolvimento do instrumento para avaliar percepção háptica. Essa versão do IAPHM é um modelo piloto. Estudos futuros determinarão confiabilidade e variações de normalidade.

Palavras-chave: percepção do tato, percepção de forma, percepção de peso, avaliação, mãos.

Currently, therapists have difficulty assessing and describing hand sensory deficits. The gold-standard tool to quantify hand tactile sensitivity is the esthesiometer. The Semmes-Weinstein esthesiometer presents nylon monofilaments of approximately the same length and of varying diameters. The diameter and length are used to control the force applied. The minimal diameter detected is used to classify the sensory function ${ }^{1}$. However, not only tactile sensitivity, but also the ability to detect weight and compressibility variations are involved in hand function.

The sensation of touch on skin is provided by mechanoreceptors in the epidermis and dermis skin layers. There are four types of mechanoreceptors: Merkel receptors detect pressure from small objects, on a frequency between 0.3 and 3.0 Hz. Meissner corpuscles detect flutter, e.g. when rubbing objects against the skin or skin movement across a surface, on a frequency between 3.0 to $40.0 \mathrm{~Hz}$. Ruffini cylinders detect pressure and stretching of the skin, on higher frequencies, between 15.0 and $400.0 \mathrm{~Hz}^{2}$. Merkel disks and Ruffini cylinders are associated with slowly adapting fibers that respond as long as the stimulus is present. Meissner corpuscles respond to stimulation with a burst of firing at the beginning and end of stimulation, therefore, they are called rapidly adapting fibers ${ }^{2}$.

\footnotetext{
'Universidade de São Paulo, Faculdade de Medicina, Curso de Fisioterapia, Laboratório de Fisioterapia e Comportamento, Sao Paulo SP, Brazil; ${ }^{2} U$ niversidade de São Paulo, Faculdade de Medicina, Departamento de Neurologia, Clínica de Distúrbios do Movimento, Sao Paulo SP, Brazil. Correspondence: Fátima Aparecida Caromano; Rua Cipotânea, 51; Cidade Universitária; 05360-160 São Paulo SP, Brasil; E-mail: caromano@usp.br Conflict of interest: There is no conflict of interest to declare. Received 03 June 2015; Received in final form 22 August 2015; Accepted 16 September 2015.
} 
The sensory system works in constant interaction with the motor system. Such interaction, which is not assessed by the Semmes-Weinstein protocol, allows the perception of objects (known as haptic perception, or stereognosis), which is achieved by the active exploration of surfaces, by moving hands $s^{3,4}$, and is fundamental in daily life activities. Therefore, tests involving haptic perception could include broader response scoring. This would allow a more detailed description of normality range (considering development ${ }^{5,6,7}$, aging ${ }^{8,9}$ and learning ${ }^{9}$ ) and the comparison between patients with sensory dysfunctions and healthy controls ${ }^{1,3,4}$.

Younger children tend to hold objects for less time than older children, and to perform less rotations for tactile examination. The sensory exploration time in children aged four months is five times longer than the visual examination time ${ }^{5}$. This fact demonstrates the intersection of different receptors from childhood to optimize perception.

One year old children can differentiate small changes (of approximately $10 \mathrm{~g}$ ) in weight ${ }^{10}$. As people get older, degradation in tactile sensory acuity is noted ${ }^{8}$. Skin loses elasticity and tactile nerve endings suffer ruptures of muscle fibers, which change in size, distribution and shape. The number of skin receptors tend to diminish ${ }^{7}$ and even previous extensive tactile experience is insufficient to preserve sensory function during aging 8.9 .

Sensory central and peripheral modifications may be associated with clinical manifestations. Grunwald ${ }^{3,4}$ related changes in the right posterior parietal cortex, the same region of tactile perception, in patients with anorexia. Hands sensory function may be altered in a great variety of neurological disorders, e.g. stroke, brachial plexus injuries, metabolic diseases. Specifically for patients with stroke, many scales have been developed to assess sensorimotor functions: Nottingham Sensory Assessment, Fugl-Meyer and Rivermead Assessment of Somatosensory Performance. However, all these scales are also classificatory ${ }^{11,12}$.

Morash et al. showed that blind individuals have better haptic performance than healthy controls, particularly when the use of multiple fingers is allowed in a tactile exploration $\operatorname{task}^{13}$. Mueller et al. showed that occupation-related long-term sensory training enhances roughness discrimination ${ }^{14}$. Conversely, recent data shows that the tactile search for changes involves less memory than visual search for changes, because the working memory has higher availability for the visual system and the haptic perception has a poorer working memory capacity ${ }^{15}$.

To better understand the normal variations, and development, aging, training, or diseases consequences on hand sensorimotor function, it is crucial to develop standardized instruments to evaluate and categorize hand haptic perception, considering different receptors functions and inputs. The objective of this study was to develop an instrument to assess hand haptic perception, generate scores and measure timed performance, and to submit the instrument to experts evaluation.

\section{METHOD}

\section{Test and manual development}

Group meetings - Five therapists discussed possible tasks to be included in the Hand Haptic Perception Instrument (HHPI), in 16 one-hour meetings. Several materials were tested until therapists reached a consensus about the best options for each task. An illustrated manual was also developed.

\section{Submission to referees}

In this stage, the instrument was evaluated by experts, who assessed the technical knowledge involved, test quality and relevance, routines for data collection, material quality and manual clearness (overall appearance, figures, technical content).

This part of the study was conducted with 30 experts (13 physiotherapists, 5 physicians, 5 occupational therapists, 4 biologists and 3 psychologists), who had obtained a minimum score of 8 (on a 0-10 scale), according to Fehring's criteria ${ }^{16}$. All experts had Ph.D. degree on health sciences, mean age 49.1 years (SD 9.3), 26 women. The mean Fehring's score was 8.5 (SD 0.6). Experts were randomly divided into 3 groups of 10. No differences on age or Fehring's score were found between the three groups.

The ten experts from group 1 analyzed the material and manual of the HHPI, and received the evaluation forms, which contained a Likert-scale questionnaire ${ }^{17}$. Each question was scored 1 for poor and 5 for very good. The corrections suggested by group 1 were discussed and incorporated in 3 meetings, by the same 5 therapists who developed HHPI (Table).

In the next stage, ten experts from group 2, analyzed HHPI and their corrections were discussed in three meetings. After the feedback from group 2, group 3 gave their suggestions, discussed on the three last meetings, in which accepted corrections were incorporated to the final version of the test.

\section{RESULTS}

\section{Experts suggestions and test corrections}

Group 1: Twelve modifications were suggested and nine of them were accepted. The structures on depression, elevation, shape recognition and barognosis domains were modified.

Group 2: Three modifications were suggested and only one was accepted. The figures of depression identification task were altered again.

Group 3: Experts recommended future studies to develop adaptations for children, older adults and patients.

\section{HHPI characteristics}

The HHPI aims to assess, score and time hand sensorimotor function, involving: Merkel receptors, which detect pressure from small objects; Meissner corpuscles, which detect 


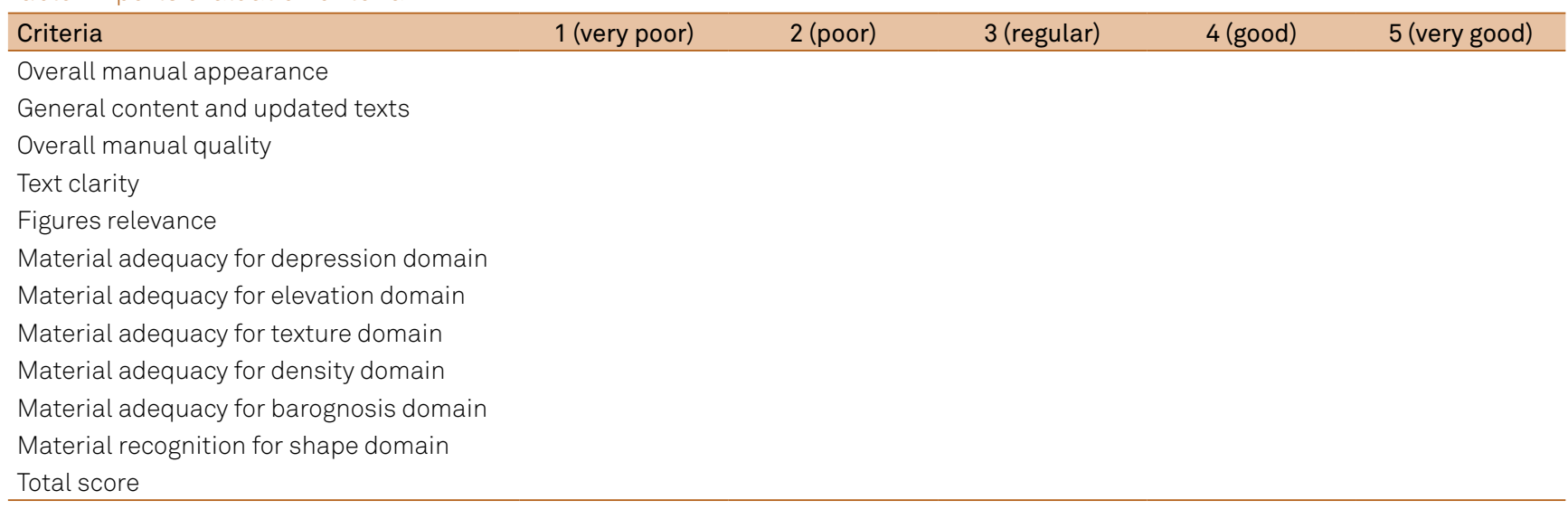

rubbing against the skin or skin moving across a surface; and Ruffini cylinders, which detect pressure and stretching. Therefore, the domains depression, elevation, texture, density, barognosis and recognition of shapes were approached.

Assessment must be conducted in quiet, well illuminated room with a table and two chairs. The examiner is positioned in front of the examinee. The examinee must be blindfolded while exploring tasks materials, except the weight from barognosis and compressibility domains. Materials consist of testing structures for each domain, evaluation chart for data collection, blindfold, pencil, rubber, A4 sheets of paper, stopwatch, camera with tripod.

We suggest this test to be filmed, because recording will allow the analysis of the exploratory movements on each task. Time measurement must be started after a verbal command authorizing the beginning of the test and stopped after the conclusion, or when the maximum time is reached (three minutes per step). Time can be measured clinically (when the test is performed), or digitally, by watching the test films.

We suggest following the sequence described on the evaluation chart to facilitate data organization and a two-minute rest between each domain. Texture domain may promote temporary skin dysesthesia, and should be the last one to be performed.

\section{Depression domain}

Purpose: To assess the ability to perceive a figure made by surface depression, to generate mental image and reproduce it by drawing on a paper and then pair it with a printed figure.

Materials: Three wooden squares $(13 \times 13 \mathrm{~cm})$ with $3 \mathrm{~mm}$ wide and deep depression figures with geometric forms (Figure 1). Task 1 is considered easy, task 2 is considered medium and task 3 , difficult.

Assessment and registration: Response analysis, according to the (1) replication, scored as 1 , if lines were correctly represented, otherwise, scored as zero; (2) proportion, scored as 1 if proportion is correct or 0 if it is incorrect and (3) location on the paper 1 if location is correct or 0 if it is incorrect. Then, the examinee has to identify the figure on a sheet with three similar figures, represented in $13 \times 13 \mathrm{~cm}$ squares. Correct answers are scored as 1 . The time must be measured and registered on each step, as additional qualitative information.

\section{Elevation domain}

Purpose: To assess the ability to perceive the position of three lines made by surface elevation (parallel, perpendicular and overlapped), to generate mental images and reproduce them by drawing.

Materials: Wooden 13 x $13 \mathrm{~cm}$ structures, containing single or double stitching lines, covered by paper and Contact $^{\circ}$ plastic (Figure 1). Difficulty levels are easy, with three double stitching parallel rows, medium, with two overlapping stitching lines and one single stitching line below and difficult, with three single stitching lines, diverging (Figure 1).

Assessment and registration: Scored according to the (1) replication, scored as 1 , if lines were correctly represented, otherwise, scored as zero; (2) proportion scored as 1 if proportion is correct or 0 if it is incorrect and (3) location on the paper, scored as 1 if location is correct or 0 if it is incorrect. Considering that this domain has three steps and that each step has one figure (easy, medium and difficult), the total score of this phase is nine. Then, the examinee has to identify the figure on a sheet with three similar figures with the same size of the tested ones. Correct answers are scored as 1 . Therefore, the total score ranges from 0 to 10 . Time is registered as qualitative information.

\section{Texture domain}

Texture, compressibility and barognosis have similar goals: The examinee must order, from left to right, three structures in accordance with the characteristics of texture, density or barognosis. The tests will take place in three steps: high, medium and low variation in textures, compressibility and weight. The last step will assess the perception of differences between structures with high/low roughness, 

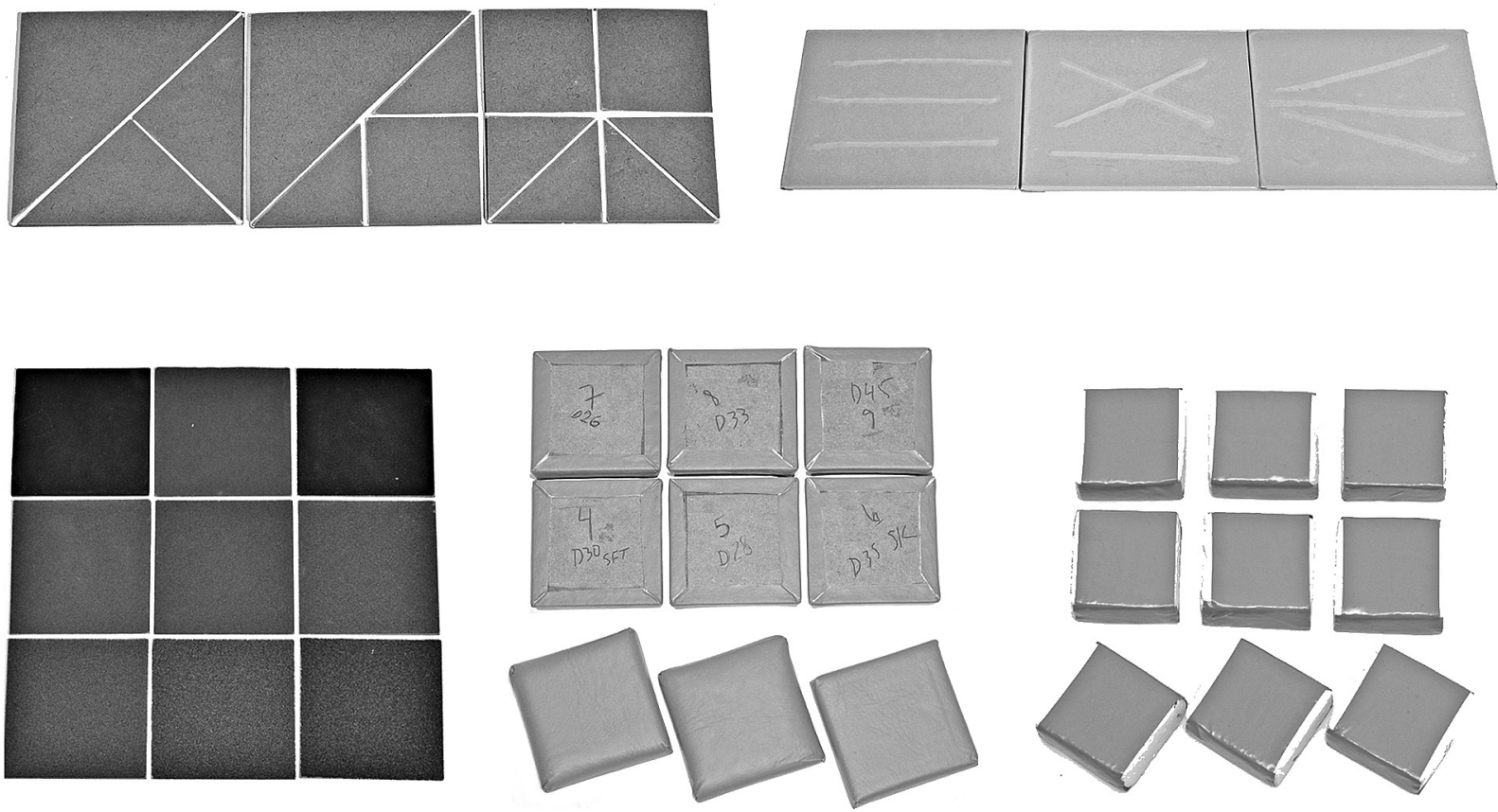

Figure 1. Above and left: figures used on depression domain (easy, medium and difficult steps). Above and right: figures of elevation domain (easy, medium and difficult). Below left: structures used on textures domain - first horizontal row: sandpapers with smoother texture, second row: sandpapers with intermediate texture and third horizontal row, rougher sandpapers. Below at center: density structures: foams of different densities covered by orange leather. Below right: Styrofoam structures of different weights, covered with yellow glossy paper, used on barognosis domain.

density or weight. The tests ends when the examinee finishes ordering the structures or when the examinee reaches the maximum time for exploration of three minutes. Correct answers are scored as 1 and incorrect, as 0 .

Materials: Nine sandpapers with different textures: 1 (water granulation 280), 2 (iron grit granulation 150), 3 (abrasive mass 60), 4 (abrasive mass 80), 5 (abrasive mass 100), 6 (abrasive mass 120), 7 (abrasive mass 150), 8 (abrasive mass 180) and 9 (abrasive mass 220). The sandpapers were glued on 13.0 x $13.0 \times 0.3 \mathrm{~cm}$ wooden boards.

Assessment and registration: The test starts with three stacked sandpapers (2, 4 and 6 or 3, 5 and 7). The examinee must organize the order of the sandpapers, from the smoother to the rougher. Each correct sequence is scored as 1 . In the second task, the examinee must distinguish between sandpapers 1,2 and 3 (smoothest ones) and the third, between 7, 8 and 9 (roughest ones). Score ranges from 0 to 3 , for each one of the three tasks. One extra point is added if the examinee can differ between the softest and roughest structures, or between distinct compressibilities (compressibility domain), or distinct weights (barognosis domain).

\section{Compressibility domain}

Materials: Nine different types of foams, with densities D13, D23, D26, D28, D28 SOFT, SOFT D30, D33, D35,
D45, SK ULTRASOFT, RIGICEL and FILTRAL were glued on $13.0 \times 13.0 \times 0.3 \mathrm{~cm}$ wooden boards, and covered with leather (Figure 1).

Assessment and registration: The examinee palpates the pieces and organizes the structures from the softest (highest compressibility) to the hardest (lowest compressibility), from left to right. The maximum time allowed is three minutes. First task includes pieces 2, 4 and 6 or 3, 5 and 7; second task includes pieces 1, 2 and 3; and the third task, pieces 7, 8 and 9 (Figure 1).

\section{Barognosis domain}

Materials: Nine styrofoam $6.5 \times 2.5 \mathrm{~cm}$ cubes, containing fishing weights from $0.6 \mathrm{~g}$ to $5.4 \mathrm{~g}$ inserted inside them, covered with Contact ${ }^{\circ}$ plastic (Figure 1).

Assessment and registration: The examinee selects three cubes according to the task difficulty level. The examinee is instructed to pick up a cube on each hand. Then, the examinee must perform aleatory movements with both hands, wrists and elbows, to increase the proprioceptive information given by the cubes in order to compare their weights. Then, the cubes must be ordered from the lightest to the heaviest, from left to right, on a maximum time of three minutes. The first task involves the pieces 2, 4 and 6 or 3, 5 and 7, the second task, pieces 1, 2 and 3 and the third task, pieces 7 , 8 and 9 (Figure 1). 


\section{Shape recognition domain}

\section{Recognition of flat shapes}

Objective: After palpating a cardboard flat geometric shape, the examinee must reproduce the figure by drawing, and then select the same figure from three options given.

Materials: Three cardboard geometric shapes (square, oval and hexagon).

Assessment and registration: After palpating the piece, the examinee is instructed to draw it. Then, the examinee must replicate the figure by drawing. The drawing is evaluated considering replication and proportion, scored as zero (wrong) or 1 (correct). Then, the examinee must locate, on a sheet of paper with three similar figures, which one represented the figure previously palpated. There are three difficulty levels (easy: square; medium: oval and difficult: hexagon).

\section{Recognition of short and tall shapes}

Objective: After palpating a short geometric shape, the examinee must find a corresponding structure among other geometric shapes with the same height, in groups of four pieces glued on a wooden board.

Materials: Twelve $0.15 \mathrm{~cm}$ (short) and twelve $1.00 \mathrm{~cm}$ (tall) wooden shapes (easy: square, triangle, rectangle, circle; medium: oval, trapezoid, hexagon, octagon and difficult: irregular polygons) glued on a wooden board (Figure 2).

Assessment and registration: The examinee must palpate the shape offered by the examiner. After that, the figure must be identified among the structures of the corresponding level (easy, medium or difficult), glued on a wooden board.

\section{DISCUSSION}

The present study aimed to develop an instrument for hand perception testing, made with affordable materials, in order to provide reproducibility on clinical and/or research conditions. Within the process of developing HHPI, the strategy of organizing groups of referees, according to Fehring's criteria, was crucial to reach a higher level of quality ${ }^{16}$. The 30 referees, distributed in three groups of 10 each, evaluated the test and gave suggestions, which resulted on the gradual improvement of the instrument.

Based on the suggestions, we tested and modified initial ideas and materials. For instance, on barognosis domain, we increased the structures weight difference. First, weights ranged from $0.2 \mathrm{~g}$ to $0.6 \mathrm{~g}$. Following the referees suggestions, we modified to a $0.6 \mathrm{~g}$ to $5.4 \mathrm{~g}$ weight range.

Regarding the tasks, firstly, we intended to use nine structures (three easy, three medium and three difficult). The referees suggested the inclusion of only three groups of structures, one of each difficulty level, otherwise testing would be too long.
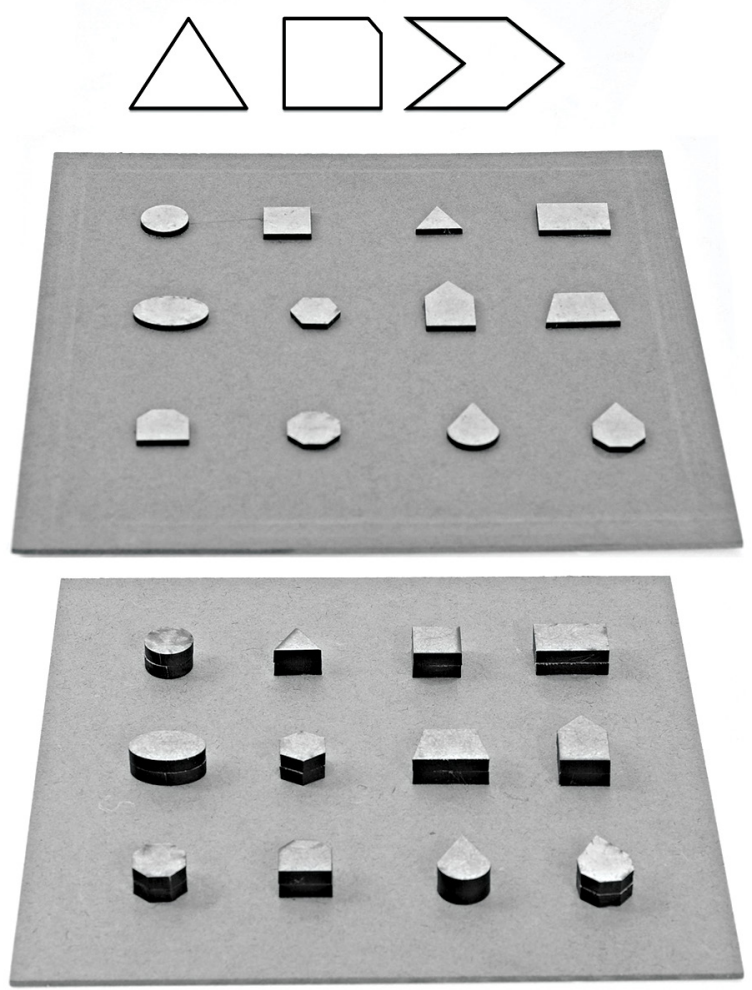

Figure 2. Shape Domain. Above: Paper Flat Figure step. Easy: triangle, average: square and difficult: arrow. In the middle, the structures used on slight elevation step. First horizontal row: easy, second row: medium and the third row: difficult. Below, structures of geometrical shapes used on great elevation step, with the same levels of difficulty of the slight elevation test.

The option to allow the manipulation and exploration of the objects without the interference of the examiner was based on a study about the role of movement explorations on the perception of length with free and restricted mobility. The authors found better results when the object was explored freely ${ }^{18,19}$.

The examiner verbal command became simpler and clearer. The verbal commands for each task were detailed explained on the manual. On barognosis domain, we added the verbal information that the examinee should move the cubes with hands, wrists and elbows movements to compare their weights ${ }^{10}$.

HHPI included the assessment of distinct neural pathways, from touching to drawing and from touching to visually identifying the figure between three possible options. We believe that this will make the test useful not only for patients with peripheral sensorimotor deficits, but also with central primary or associative sensorimotor disorders ${ }^{20}$. Besides, timed performance, which is additional qualitative measure, will enrich the discussion of motor strategies. Filming the tasks will provide complementary information for such analysis, allowing the description of the exploratory strategies. HHPI can be used to complement the current gold-standard tests, which focus specifically on tactile, thermic, vibratory and pain perception. 
The present paper aimed to present a new instrument, and future studies will describe normality variations on healthy population, clarifying differences on haptic performance, according to dexterity, development, aging, previous hand training, motor strategies. We also intend to perform subsequent reliability and validity analyses.
In conclusion, we developed a new instrument, named HHPI, which evaluates hand distinct sensory receptors functions and generates scores and timed performance. The instrument testing process, with groups of referees, allowed the analysis of HHPI adequacy to measure haptic perception.

\section{References}

1. Weirte J, Moortgat P, Truijen S, Maertens K, Lafaire C, De Cuyper $L$ et al. Interrater and intrarater reliability of the Semmes-Weinstein aesthesiometer to assess touch pressure threshold in burn scars. Burns. 2015;41(6):1261-7. doi:10.1016/j.burns.2015.01.003

2. Johansson F. Coding and use of tactile signals from the fingertips in object manipulation tasks. Nature Rev Neurosci. 2009;10:345-59. doi:10.1038/nrn2621

3. Grunwald M, John M. German pioneers of research into human haptic perception. In: Grunwald M, editor. Human haptic perception: basics and applications. Boston: Basel; 2008. p. 15-39.

4. Grunwald M, Weiss T, Assmann B, Ettrich C. Stable asymmetric interhemispheric theta power in examinees with anorexia nervosa during haptic perception even after weight gain: a longitudinal study. J Clin Experim Neuropsychol. 2004;26(5):608-20. doi:10.1080/13803390409609785

5. Streri A, Spelke E S. Haptic perception of objects in infancy. Cognitive Psychol. 1988;20(1):1-23. doi:10.1016/0010-0285(88)90022-9

6. Jao RJ, James TW, James KH. Multisensory convergence of visual and haptic object preference across development. Neuropsychologia. 2014;56:381-92. doi:10.1016/j.neuropsychologia.2014.02.009

7. Klevberg GL, Anderson DI. Visual and haptic perception of postural affordances in children and adults. Human Movem Sci. 2002;21(2):169-86. doi:10.1016/S0167-9457(02)00100-8

8. Guest S, Mehrabyan A, Ackerley R, McGlone F, Phillips N, Essick G. Tactile experience does not ameliorate age-related reductions in sensory function. Exp Aging Res. 2014;40(1):81-106. doi:10.1080/0361073X.2014.857563

9. Reuter M, Voelcker-Rehage C, Vieluf S, Godde B. Touch perception throughout working life: effects of age and expertise. Exp Brain Res. 2012;216(2):287-97. doi:10.1007/s00221-011-2931-5

10. Molina M, Jouen F. Weight perception in 12-month-old infants. Infant Behav Dev. 2003;2(1)6:49-63. doi:10.1016/S0163-6383(02)00168-6
11. Lincoln NB, Jackson JM, Adams SA. Reliability and revision of the Nottingham Sensory Assessment for stroke patients. Physiotherapy. 1998;84(8):358-65. doi:10.1016/S0031-9406(05)61454-X

12. Lin JH, Hsieh IP, Shen CF, Hsieh CL. Psychometric properties of the sensory scale of the Fugl-Meyer Assessment in stroke patients. Clin Rehabil. 2004;18(4):391-7. doi:10.1191/0269215504cr737oa

13. Morash VS, Pensky AEC, Tseng STW, Miele JA. Effects of using multiple hands and fingers on haptic performance in individuals who are blind. Perception. 2014;43(6):569-88. doi:10.1068/p7712

14. Mueller S, Winkelmann C, Krause F, Grunwald M. Occupation-related long-term sensory training enhances roughness discrimination but not tactile acuity. Exp Brain Res. 2014;232(6):1905-14. doi:10.1007/s00221-014-3882-4

15. Yoshida T, Yamaguchi A, Tsutsui H, Wake T. Tactile search for change has less memory than visual search for change. Atten Percept Psychophys. 2015;77(4):1200-11. doi:10.3758/s13414-014-0829-6

16. Fehring RJ. Validation diagnostic labels: standardized methodology. In: Hurley ME. Classification of nursing diagnoses: proceedings of the sixth conference of North American Nursing Association. St. Louis: Morby; 1986. p. 183-90.

17. Likert R. A technique for the measurement of attitudes. Arch Psychol. 1932;22(140): 55 .

18. Lobo L, Travieso D. El patrón de exploración modula la percepción de longitudes a través del tacto dinamico. Psicothema. 2012;24(1):55-61.

19. Parianen Lesemann FH, Reuter EM, Godde B. Tactile stimulation interventions: influence of stimulation parameters on sensorimotor behavior and neurophysiologic correlates in healthy and clinical samples. Neurosci Biobehav Rev. 2015;51:126-37. doi:10.1016/j.neubiorev.2015.01.005

20. Diego C, Puig S, Navarro X. A sensorimotor stimulation program for rehabilitation of chronic stroke patients. Restor Neurol Neurosci. 2013;31(4):361-71. doi:10.3233/RNN-120250 